\title{
The therapeutic use of botulinum toxin in cervical and maxillofacial conditions
}

\author{
Abstracted from \\ Ihde SK, Konstantinovic VS. \\ The therapeutic use of botulinum toxin in cervical and maxillofacial conditions: an evidence-based review. \\ Oral Surg Oral Med Oral Pathol Oral Radiol Endod 2007 Aug;104 (2): e-publication \\ Address for correspondence: Stefan K. A. Ihde, CH-8737 Gommiswald, Switzerland E-mail dr.ihde@blu
}

\section{Question: Is botulinum toxin safe to be used prophylactically in people undergoing dental implant therapy?}

Data Sources Medline, Cochrane Library and bibliographies of identified articles.

Study selection Studies of high methodological quality (systematic reviews or randomised controlled trials; RCT) were selected if considered use of botulinum toxin (BTX) either prophylactically or therapeutically, as an adjunct to dental implant therapy for temporomandibular disorders or for other maxillofacial conditions such as bruxism, masseteric hypertrophy, oromandibular dystonia or cervical dystonia.

Data extraction and synthesis Only studies with high levels of evidence were evaluated. Four RCT met the search criteria in the area of cervical dystonia and chronic facial pain.

Results No RCT were identified that evaluated dental implant therapy along with use of BTX. Four RCT did meet the search criteria in the area of cervical dystonia and chronic facial pain. People who had cervical dystonia exhibited significant improvements in baseline functional, pain and global assessments compared with placebo, whereas individuals with chronic facial pain improved significantly compared with placebo in terms of pain.

Conclusions During the period of study, no references were found evaluating use of BTX in dental implantology. Nevertheless, it appears relatively safe and effective in treating cervical dystonia and chronic facial pain associated with masticatory hyperactivity.

\section{Commentary}

BTX has been used as a therapeutic agent since 1977 to treat several medical conditions. Previously, it was recognised as a toxic agent. There are eight serological types of which types A and B are the most commonly used therapeutically. The use of BTX has been extended to include the fields of facial aesthetics and more recently cancer research. ${ }^{1}$ There are, however, only a few good quality RCT that have looked at the clinical effectiveness and safety of BTX.

Four products that contain BTX are licensed in the UK: Botox (Allergan Ltd, Marlow, Bucks), Dysport (Ipsen Ltd, Slough, Berks), NeuroBloc (Eisai Ltd, Hammersmith, London) and Vistabel (Allergan Ltd, Marlow, Bucks). The UK's Medicine and Healthcare Products Regulatory Agency has licensed the use of BTX in focal spasticity, some types of spasm and excessive sweating of the armpits. Vistabel is indicated only for treatment of vertical frown (glabellar) lines between the eyebrows, where the patient suffers a severe psychological effect from their facial appearance.

This review paper shows that BTX is relatively safe and effective compared with placebo in the treatment of cervical dystonia $(n=3$, all using BTX type B) and maxillofacial conditions ( $\mathrm{n}=1$, using BTX type A). It extends these findings as a guide for treating dental implant patients prophylactically in immediate loading protocols. The review shows that BTX is safe and efficacious in the therapeutic use of maxillofacial conditions and cervical dystonia.

Using the methods described in this review paper, a Medline search identified 14 other RCT that used BTX in the treatment of cervical dystonia. This included both BTX type A and type B and placebo-controlled studies, dose-dependent studies and studies comparing BTX from two manufacturers (Botox and Dysport). It is therefore unclear if the search criteria in this review paper only included placebo-controlled studies of type B in the treatment of cervical dystonia. The Medline search identified a second review on myofacial pain which was published recently. ${ }^{2}$ Further placebo-controlled studies with high methodological quality on the efficacy and safety of BTX in maxillofacial conditions are required.

Taken together, current evidence shows that treatment with BTX produces an effect within 1 week, peaking with a maximum response around 1 month, and lasting between 3 and 6 months. There is a lack of evidence, still, to show the effects of repeated BTX injections and any long-term adverse effects associated with its use. Further long term studies are required to understand effects of BTX on nerve terminals, and any clinical implications associated with its use.

BTX is increasingly being used in medicine and dentistry, and has been linked with a few reversible adverse effects. At present there are no studies that demonstrate any beneficial or adverse effects of BTX use in dental implantology. BTX procedures are technique-sensitive and may be aided by the use of electromyography. Further good quality studies are required to understand the properties, clinical efficacy and any associated long-term adverse effects.

Kushal Gadhia and Damien Walmsley School of Dentistry, University of Birmingham, St Chad's Queensway, Birmingham, UK

1. Ansiaux R, Gallez B. Use of botulinum toxins in cancer therapy. Expert Opin Investig Drugs 2007: 16: 209-218.

2. Guarda-Nardini L, Manfredini D, Salamone M, Salmaso L, Tonello S, Ferronato G. Efficacy of botulinum toxin in treating myofascial pain in bruxers: a controlled placebo pilot study. Cranio 2008; 26: 126-135.

Evidence-Based Dentistry (2009) 10, 53. doi:10.1038/sj.ebd.6400654 